\title{
Reflexions-Pulsoximetrie - Untersuchungen mit eigenem Mess-System
}

König, Volker ; Ullrich, Georg J ; Faisst, Karin ; Jørgensen, Jan Stener ; Huch, Renate ; Huch, Albert

DOI: https://doi.org/10.1515/bmte.1992.37.s2.39

Posted at the Zurich Open Repository and Archive, University of Zurich

ZORA URL: https://doi.org/10.5167/uzh-155427

Journal Article

Published Version

Originally published at:

König, Volker; Ullrich, Georg J; Faisst, Karin; Jørgensen, Jan Stener; Huch, Renate; Huch, Albert (2009). Reflexions-Pulsoximetrie - Untersuchungen mit eigenem Mess-System. Biomedizinische Technik. Biomedical engineering, 37(Erg. Bd.):39-40.

DOI: https://doi.org/10.1515/bmte.1992.37.s2.39 


\title{
Reflexions-Pulsoximetrie - Untersuchungen mit eigenem Mess-System
}

\author{
Volker König, Georg J. Ullrich, Karin Faisst, Jan Stener Jørgensen, Renate Huch und Albert Huch
}

\section{Klinik und Poliklinik für Geburtshilfe, Universitätsspital Zürich}

Die nichtinvasive Ueberwachung der arteriellen Sauerstoffsättigung durch pulsoximetrische Transmissionsmessung ist heute ein in der klinischen Routine etabliertes Verfahren. Dagegen sind pulsoximetrische Reflexionsmessungen noch im experimentellen Stadium, obwohl sie in gewissen Fällen entscheidende Vorteile bieten:

- Messung ist möglich an zentraleren Stellen, an denen ein Versagen infolge Zentralisation des Kreislaufes weniger zu erwarten ist.

- Messung ist möglich an Stellen, die einer Transmissionsmessung unzugänglich sind (z.B. sub partu Messungen am Kind).

Sie haben demgegenüber allerdings auch Nachteile:

- Die Befestigung des Sensors ist schwieriger.

- Es treten zusätzliche physikalische und physiologische Effekte auf, die die Kalibrierung der Messung beeinträchtigen können.

Als die wesentlichsten dieser störenden Effekte sind anzusehen:

- Licht kann bei nicht sachgerechter Konstruktion des Sensors direkt vom Sender oder durch direkte Reflexion an den obersten Hautschichten in den Empfänger gelangen ("Direktes Licht" DL).

- Die absorptiv wirksamen Lichtwege für rotes und infrarotes Licht im Gewebe können unterschiedlich sein und deshalb wird von beiden Wellenlängen nicht das gleiche Kapillarbett durchstrahlt.

- Durch Bewegungen (z.B. des Herzens bei einer Messung auf der Brust oder durch die Nähe stark pulsierender Arterien) kann Gewebe je nach innerer Festigkeit rein mechanisch zu "pulsatilen" Dickenänderungen angeregt werden. Da die Signaländerung durch die arterielle Pulsation maximal $1 \%$ beträgt, können derartige pulsatile Dickenänderungen von nur einigen $\mu \mathrm{m}$ die Kalibrierung bereits merkbar beinflussen.

\section{Das Mess-System.}

Für Untersuchungen der Zusammenhänge bei pulsoximetrischen Reflexionsmessungen wurden in der Perinatalphysiologischen Forschungsabteilung der Klinik für Gcburtshilfe eine Reihe von Sensoren entwickelt. Die berichteten Ergebnisse wurden mit einem radialsymmetrischen Sensor von $21 \mathrm{~mm}$ Durchmesser erhalten. Im Zentrum befindet sich ein Chin mit 2
Durchmesser von $17 \mathrm{~mm}$ sind als Empfänger 6 Photodioden (Siemens BPX90) angebracht.

In Anlehnung an Entwicklungen von Forstner und Faust wurde die Mess-Elektronik ausgeführt. Diese steuert die LEDs im Zyklus Rot-Dunkel-InfrarotDunkel, mit einer Zyklus-Frequenz von normalerweise $400 \mathrm{~Hz}$. Die LED-Ströme werden dabei so eingestellt, dass die gemessenen Photoströme für beide Wellenlängen etwa gleich sind. Nach veränderbarer Verstärkung und einer Sample-and-Hold-Stufe wird der Dunkelstrom subtrahiert. Die pulsunabhängigen DCSignale und die pulssynchronen AC-Signale gelangen über eine ADC-Karte in einen IBM 386-Computer. Ein PASCAL-Programm liest diese Werte über DMA ein und berechnet nach bestimmten Algorithmen die Pulsfrequenz, die Perfusion und die Sauerstoffsättigung. Diese Werte werden über maximal 9 Herzzyklen gemittelt und in jeder Sekunde in ein File eingeschrieben. Der Display zeigt den zeitlichen Verlauf dieser Werte, sowie wahlweise die ReflexionsSignale (Einstell-Phase) oder die Häufigkeitsverteilungen der gemessenen Sauerstoffsättigungen und Pulsfrequenzen oder der Perfusionswerte (Mess-Phase). Abb.1 zeigt einen solchen Display mit einer Auswertung von 0:15 bis 3:15 Minuten.

Für die Anwendung an Patienten wurde das gesamte Mess-System durch Trenntrafos und DC-DC-Wandler vom Netz getrennt. In einige Sensoren wurden Heizdrähte sowie zwei NTC-Widerstände zur Temperaturmessung integriert. Durch eine hochstabile Heizelektronik kann damit das Gewebe im Messbereich aufgeheizt werden. Geeignete Versuche sollen zeigen, ob die hierdurch induzierte grössere Perfusion zu einem höheren und stabileren Mess-Signal fuihrt.

\section{Berechnung der Sauerstoffsättigung.}

Aus den vier Werten pulsunabhängiges Signal (DC), pulssynchrones Signal (AC), jeweils für das rote (rot) und infrarote (ifr) Licht, ergibt sich der Messwert

$$
R=\left(I_{A C} / I_{D C}\right)_{\text {rot }} /\left(I_{A C} / I_{D C}\right)_{\text {ifr }} \text {. }
$$

Eine genauere Analyse des Absorptionsvorganges zeigt, dass noch die folgenden Korrekturen in die Messung eingehen können: 


$$
\mathrm{C}_{1}=\mathrm{d}_{\mathrm{rot}} / \mathrm{d}_{\mathrm{ifr}} \text {. }
$$

dem Verhailtnis der effekliven Lichtwege für rot und infrarot, und

$$
\mathrm{C}_{2}=\left(1+\mathrm{I}_{\mathrm{DL}} / \mathrm{I}_{\mathrm{DC}}\right)_{\mathrm{rot}} /\left(1+\mathrm{I}_{\mathrm{DL}} / \mathrm{I}_{\mathrm{DC}}\right)_{\mathrm{ifr}} \text {. }
$$

dem Verhälınis des direkten Lichtanteils DL zum DCAnteil für beide Wellenlängen. Aus geometrischen Gründen sind diese beiden Faktoren für Transmissionsmessungen nahezu $=1$, sie können aber im Reflexionsfall einen Einfluss auf die Berechnung der Săttigung ausüben.

Die gesuchte Sauerstoffsättigung ist nun eine Funktion von R bzw. von R' und kann hieraus in erster Näherung mit dem Lambert-Beerschen-Gesetz (LBG) bei Kenntnis der Absorptionskoeffizienten berechnet werden. Es ist aber bekannt, dass die Anwendung des LBG, besonders bei niedrigen Sättigungen, inkorrekte Werte liefert und deshalb eine experimentelle Kalibrierung notwendig ist. Wir haben für unsere Messungen zunächst das LBG zugrunde gelegt und versuchen, eine Korrektur durch Vergleichsmessungen mit einem MINOLTA Pulsox8 Transmissions-FingerPulsoximeter vorerst im Sättigungsbereich von ca. 85$100 \%$ durchzuführen.

\section{Messungen an verschiedenen Probanden.}

Als erste Untersuchungen wurden Messungen an gesunden Probanden ausgeführt: zuerst Atmung von normaler Luft, dann für 10 Minuten ein Gasgemisch mit ca. 15\% Sauerstoff und danach wieder normale Luft. Als Monitor und Vergleichsmessung diente ein MINOLTA Pulsox8 Transmissions-Finger-Pulsoximeter. Dabei wurden zunächst die folgenden Punkte untersucht:

- Messung an verschiedenen Probanden mit Wiederholungen an verschiedenen Tagen.

- Befestigung der Sonde mit EKG-Klebering oder mit Klebeband über dem Sensor.

- Applikation am Sternum und an der Stirn.

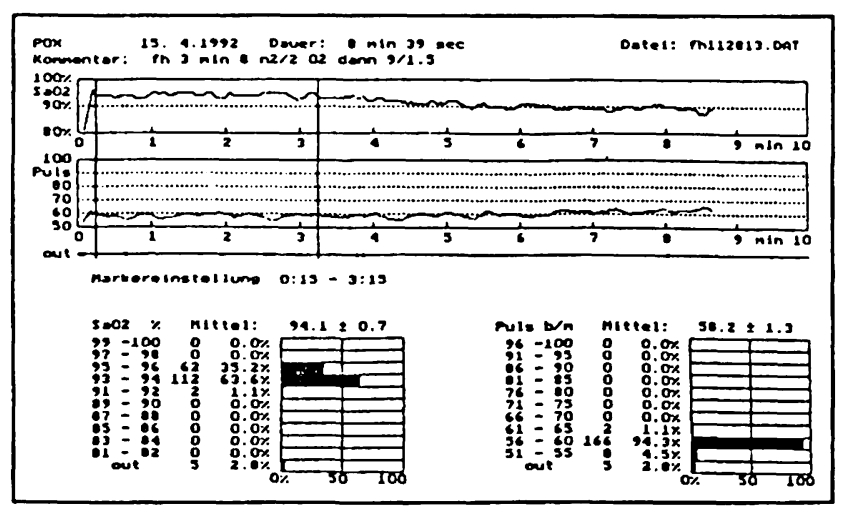

Abb.1: Computer-Ausdruck für Messung an der Stirn. Zuerst Atmung von Luft, nach 3 Minuten Gemisch mit 15\% Sauerstoff. Auswertebereich 0:15-3:15 Min.
Diskussion der Messungen.

Ein Beispiel einer solchen Messung an der Stirn zeigt Abb.2. Aufgetragen ist die Differenz zwischen Transmissions- und Reflexionsmessung gegen die Transmissionsmessung. Aus der Gesamtheit aller Messungen ergeben sich die folgenden Tatsachen:

- Der Mittelwert der Reflexionsmessungen weicht von den als Standard angeschenen MINOLTA-Werten um 4.5\% ab. Dies ist auf die Anwendung des unkorrigierten LBG für unsere Auswertungen zurückzuführen.

- Die ca. 20 Minuten dauernden Messungen an einem Probanden zeigen in sich Abweichungen von maximal $\pm 0.7 \%$, mit einer mittleren Streuung der Einzelwerte von $1.4 \%$, verglichen mit der Streuung der MINOLTAWerte von $0.8 \%$. Die Abweichungen von Proband zu Proband, auch bei einer neuerlichen Messung an demselben Probanden (mit neuer Befestigung der Sonde) liegen im Bereich von bis zu $\pm 2.6 \%$. Gemäss den obigen Bemerkungen können diese Unterschiede auf verschiedene Lichtwege für die zwei Wellenlängen $\left(C_{1}\right)$, auf Autreten von direktem Licht $\left(\mathrm{C}_{2}\right)$ an verschiedenen Applikationsorten oder mechanische pulsatile Bewegungen zurückzuführen sein.

- Die Mittelwerte für die beiden Befestigungsmethoden entsprechen den Mittelwerten für alle Messungen.

- Das Gleiche gilt für die Applikationsorte Sternum und Stirn.

Diese Messungen zeigen, dass mit der ReflexionsMethode sehr wohl zeitliche Aenderungen der Sauerstoffsättigung genau gemessen werden können, eine Bestimmung des absoluten Wertes hingegen um $\pm 2.6 \%$ ungenau sein $k a n n$. Ob die verschiedenen Weglängen des Lichtes, Auftreten von Direktlicht oder weitere Effekte für dieses Verhalten verantwortlich sind, sollen weitere Untersuchungen, eventuell unter Verwendung einer Blutgasanalyse, zeigen. Ausserdem werden diese Versuche unter Einbezug des Effektes der Heizung des Gewebes im Bereich der Mess-Stelle fortgesetzt.

Literatur: Bei den Autoren erhältlich.

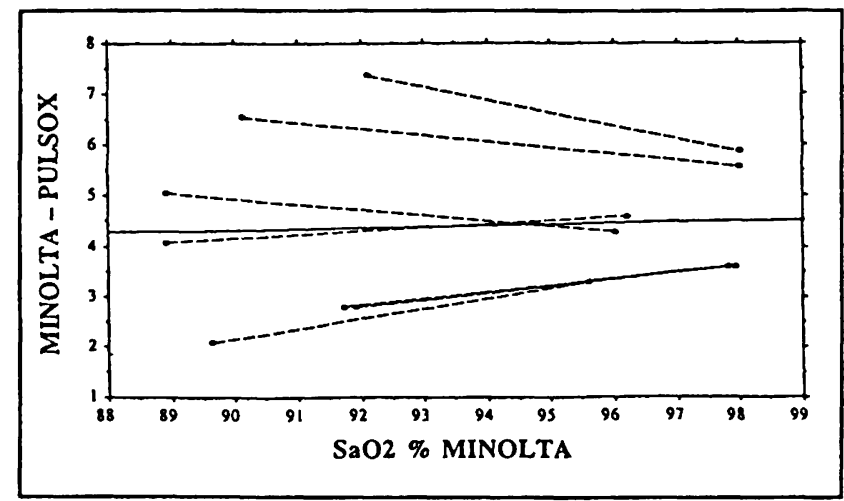

Abb.2: Für 7 Probanden Messung an der Stirn. Abszisse: Sătligung MINOLTA, Ordinate: Differenz zwischen MINOLTA und unserem Mess-System. Durchgezogene Linie: Mittelwert aller Messungen. 